\title{
The application of the median filter in image restoration
}

\author{
Fengming Zhou ${ }^{1, a}$, Weilan Wang ${ }^{2, b}$ \\ ${ }^{1,2}$ Department of Math and Computer Science, Northwest University for Nationalities, Lanzhou City, \\ China \\ a593349546@qq.com, ${ }^{b}$ correspondence author: wangweilan@xbmu.edu.cn
}

Keywords: Image Restoration; Median Filtering; Template Size.

Abstract. Image filtering is an important part in the process of image preprocessing, such as get rid of the noise from image. This paper introduces the application of median filter in image restoration, and the purpose is to repair the contains a lot of tiny crack in images. By comparing all kinds of filter, median filter has better ability to repair; To compare various templates of median filtering, square template have better repair effect; By choosing different template in size, it can get a different repair effect. Finally, the results of the experiments show that it can obtain good results when we are adopt to the median filtering for repairing the crack of the images.

\section{Introduction}

Image restoration technology is currently a hot research topic in image processing, which has important application value in the protection of cultural relics and in the targeted image erasure and so on. In this paper, the median filter is adopted to image restoration, and the filter consists of linear filtering and nonlinear filtering. Linear filtering method usually has low-pass characteristic and the image edge information corresponding to the high frequency signal, so the linear filtering method would result in a blurred image edge. Median filter is a kind of less edge fuzzy nonlinear filtering method, not only can remove or reduce random noise and impulse noise, but also retains the image edge information good [1]. In literature [2], this paper proposes a combination of median filtering and image restoration method based on the curvature diffusion, and considering the geometry information of the image itself, the repair on the geometric features of images have higher accuracy.

We consider the problem of image itself, when the tiny cracks in image processing. It is more effective that using the method of simple and fast to repair processing. Therefore, we put forward the way of the median filtering for image restoration.

\section{Experimental Method}

The Effect Of Different Filter To Repair Damaged Pieces. In image restoration, we usually use around the damaged area of known points information, with linear or nonlinear extrapolation method to calculate the value of the point, in order to achieve the purpose of repairing damaged area. And median filtering is a kind of nonlinear, it is the use of adjacent pixels of known points to get damaged pixels pixel values.

The average filtering is a linear filter and has a similar function with median filtering, can eliminate the noise in the image, but the average filtering in removing noise also break down the details in the image at the same time, the median filter in removing image noise can protect the edges of the image at the same time, make its be not fuzzy. Using the same template in the size of the average filtering and median filtering for image restoration, and the results, as shown in Fig. 1.

From Fig.1b, Fig.1c , It is obvious that the outcomes of using the average filtering restoration have boundary, the result of repairing for damaged areas is bad; However, the result that using the median filter not only has fairly good repair effect, but also can better protect the boundary. So the median filter can be used for image damaged crack repair work. 


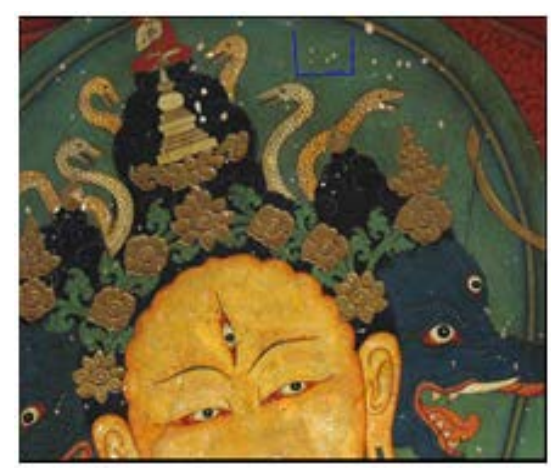

a. The original image

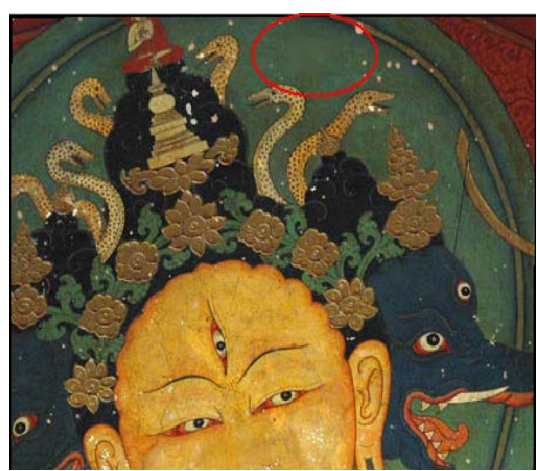

b. Median filtering effect

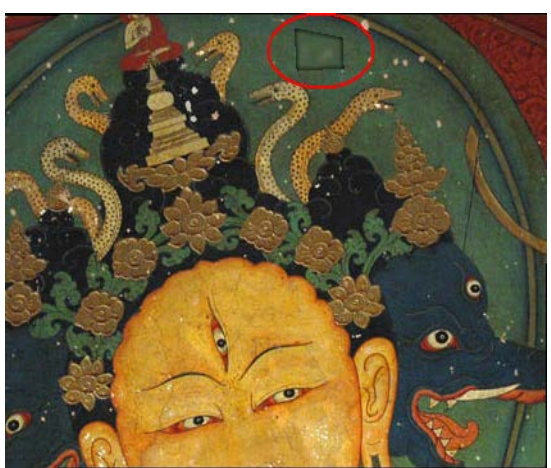

c. Average filtering effect

Fig. 1.Different filter on the image processing effect

The Relationship Between The Different Templates And Repair Damaged Pieces. On image restoration of different templates, the effect is different. Commonly used template has linear templates with square template.

A. Linear template. Set a fixed point of $P(i, j)$, template $S$, template length is $N, S$ and $N$ is odd, $S$ $=\left[\begin{array}{llll}1 & 1 & 1 & 1\end{array} \ldots .\right.$. . Using templates $\mathrm{S}$ convolution computation of target area, as shown in Fig. 2 .

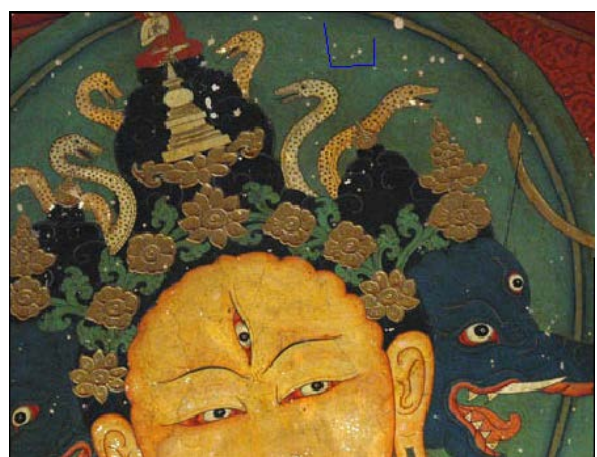

a. The original image

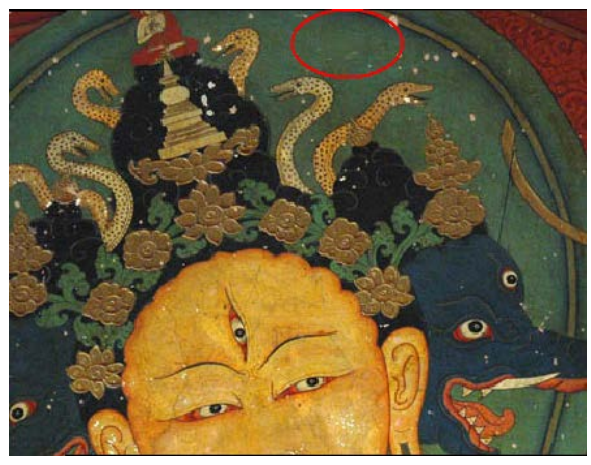

b. Effect image

Fig. 2.The repair effect of the linear template

B. Square template. Set a fixed point for $\mathrm{P}(\mathrm{i}, \mathrm{j})$, template $\mathrm{S}$, width of the template is $\mathrm{N}$, and $\mathrm{N}$ is odd, $\mathrm{S}=\left[\begin{array}{lllllll}1 & 1 & 1 & \ldots, & 1 & 1 & 1 \ldots, \ldots\end{array}\right]$. Centering on the $\mathrm{P}$, the size of square template is $\mathrm{N} \times \mathrm{N}$ and multiplication with the points under square template, the effect of the gray value is $u 1, u 2, u 3, \ldots$. . Order the convolution results, and the median will be calculated in the value assigned to point $\mathrm{P}$, and then traverse the entire area, get the final result, as shown in Fig. 3.

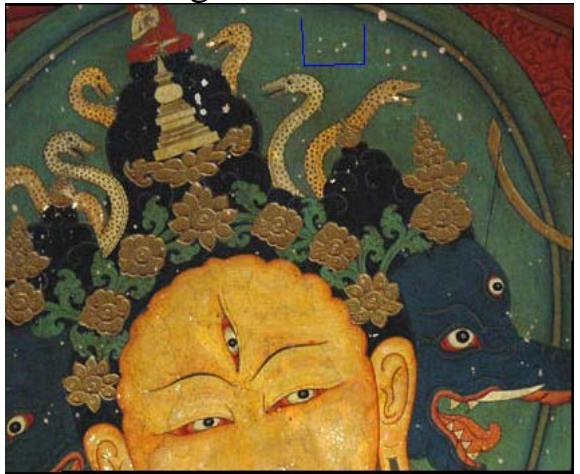

a. The original image

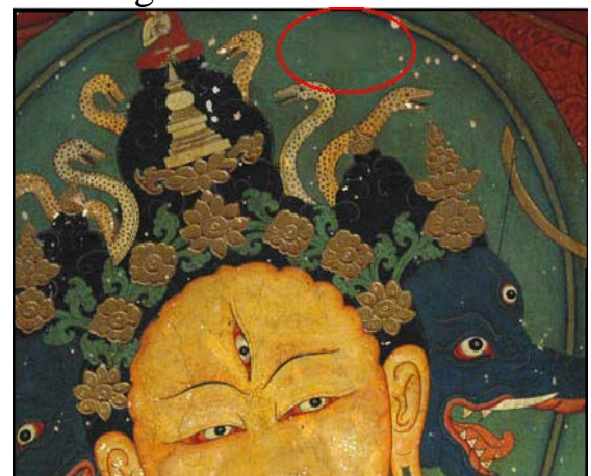

b. Effect image

Fig. 3.The square template rendering

Comparing Fig. 2 and Fig. 3, it can be seen in Fig. 3b is better than Fig. 2b about repair effect. To be linear template, the obtained information of point $\mathrm{P}$ Where does it come from right and left of the point $\mathrm{P}$, it did not consider other six point information that the neighborhood point of point $\mathrm{P}$, So the values obtained using linear template have larger deviation. However, the square template take into account the information that the eight neighborhood of point P, So the repair effect is better than linear template. Therefore, taking the square template of median filtering to repair the damage area. 
The Relationship Between The Different Size Of Templates And Repair Damaged Pieces. Using the filter for image processing has a great influence when select different size of the template on the repair results.

A. The different crack width of $3 \times 3$ template for image processing, as shown in Fig. 4 .

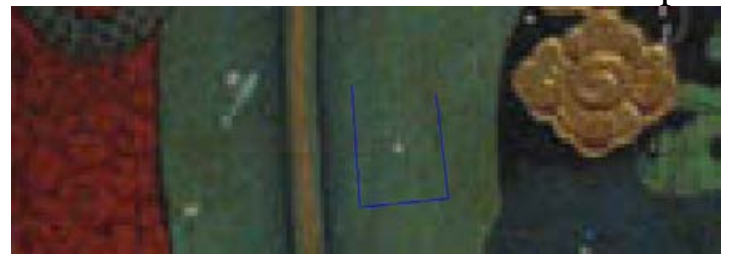

a. Two pixel width

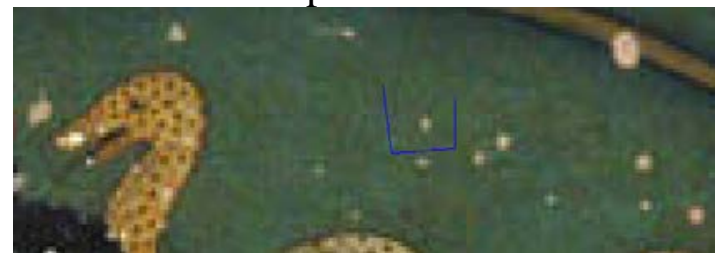

b. Three pixel width

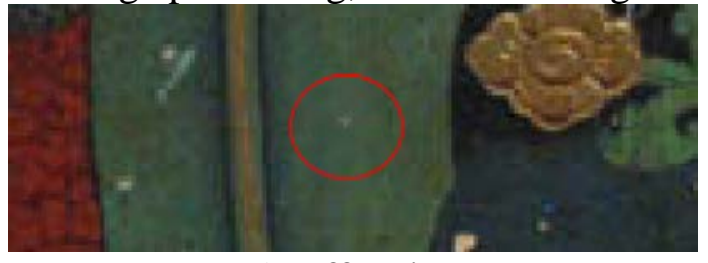

a_1. Effect image

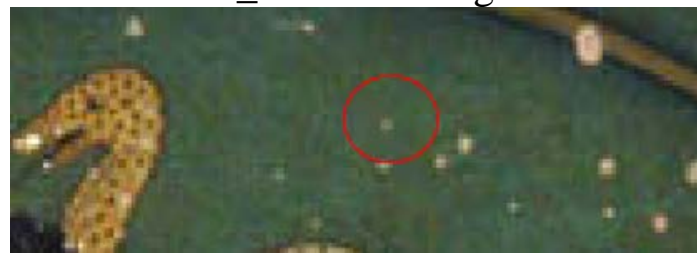

b_1.Effect image

Fig. $4.3 \times 3$ template

Experimental results show that when the image crack width is greater than 2 pixels, the size of the template with $7 \times 7$, repair bad results.

B. The different crack width of $5 \times 5$ template for image processing, as shown in Fig. 5.

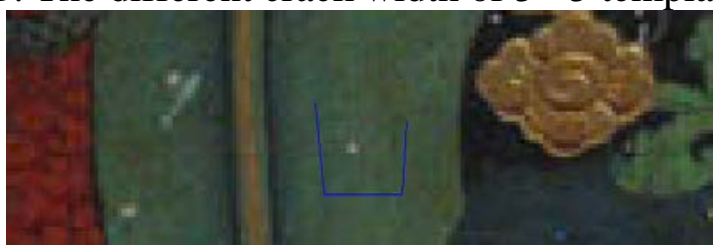

a. Two pixel width.

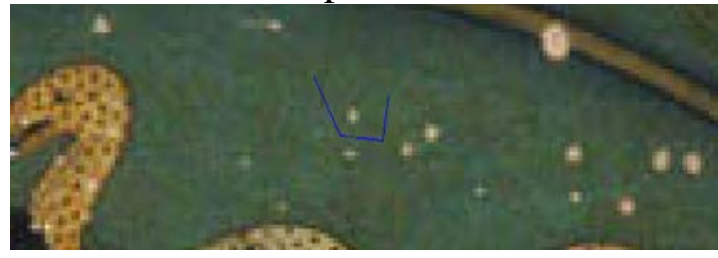

b. Three pixel width

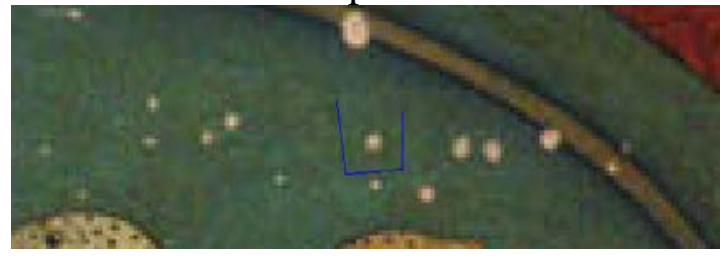

c. Four pixels width

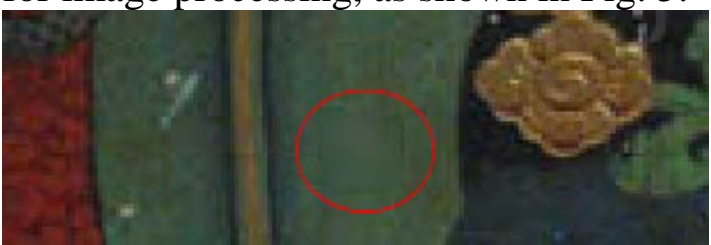

a_1. Effect image

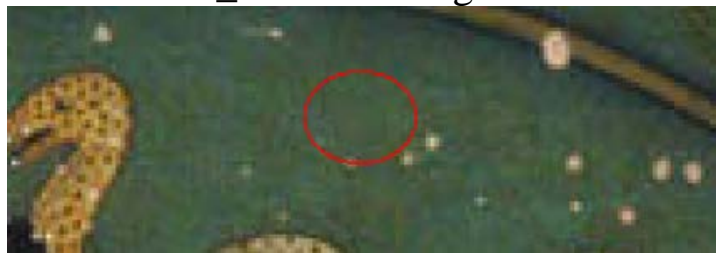

b_1. Effect image

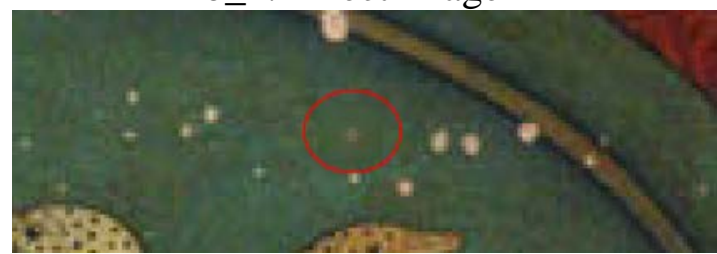

c_1. Effect image

Fig. 5.5×5 template

Experimental results show that when the image crack width is greater than 3 pixels, the size of the template with $7 \times 7$, the results is bad.

C. The different crack width of $7 \times 7$ template for image processing, as shown in Fig. 6 .

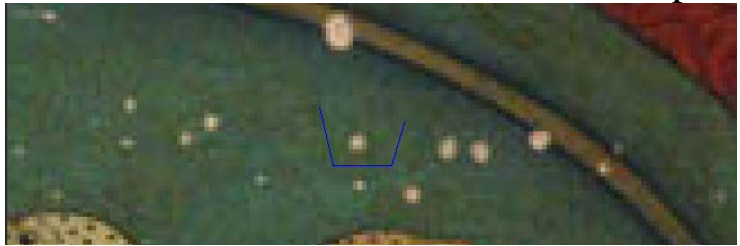

a. Four pixels width

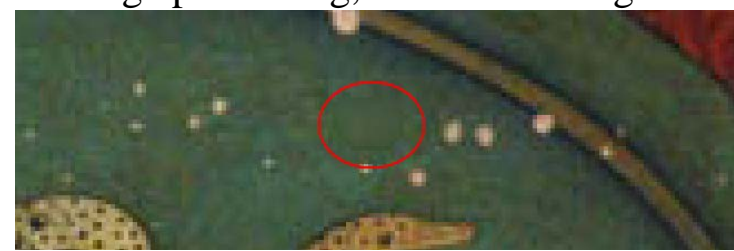

a_1, Effect image 


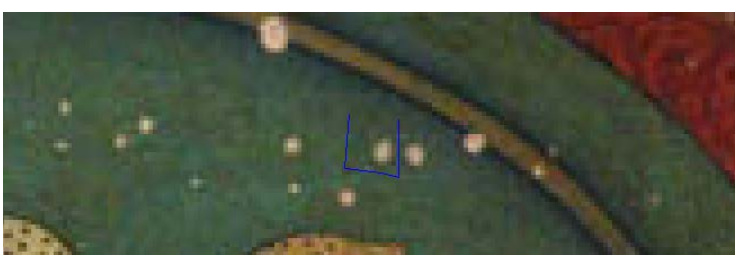

b. 5 pixels width

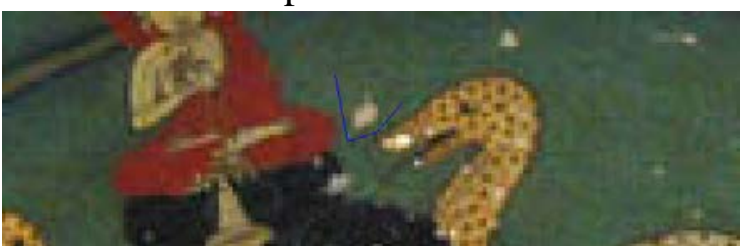

c. 6 pixel width

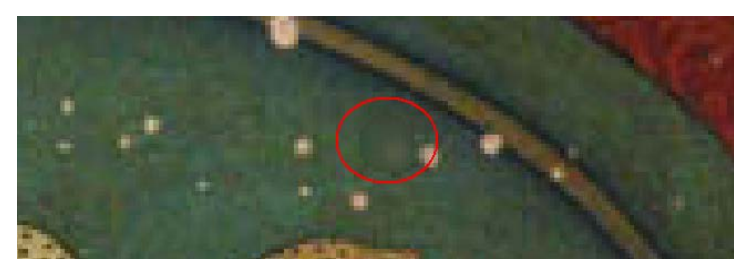

b_1. Effect image

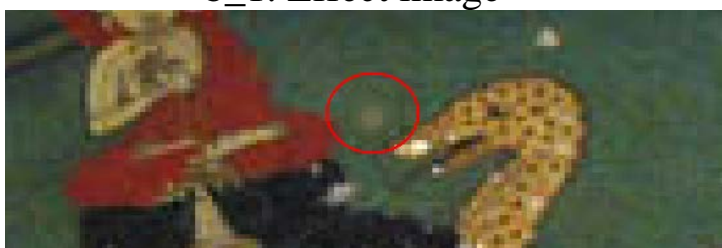

c_1. Effect image

Fig. $6.7 \times 7$ template

Experimental results show that when the image crack width is greater than 5 pixels, the size of the template with $7 \times 7$, has a bad results.

D. The different crack width of $9 \times 9$ template for image processing, as shown in Fig. 7.

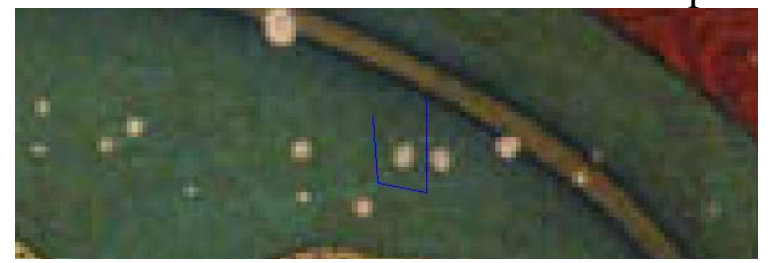

a. 5 pixels width

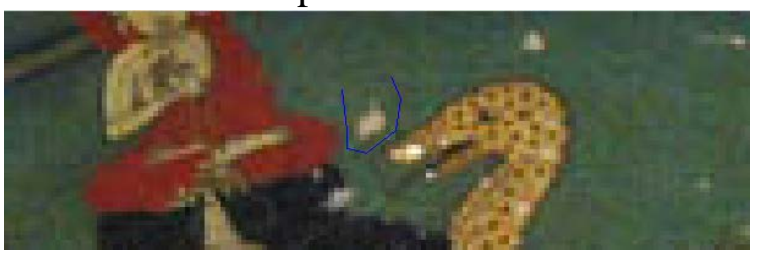

b. 6 pixel width

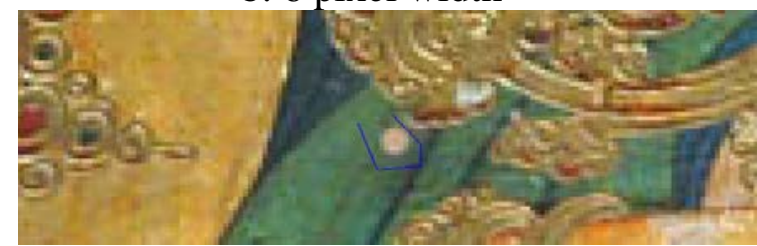

c. 7 pixel width

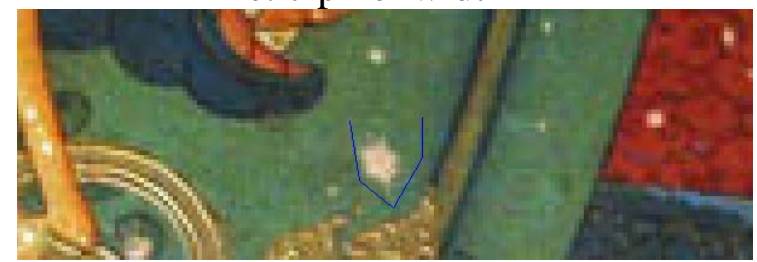

d. Eight pixels width

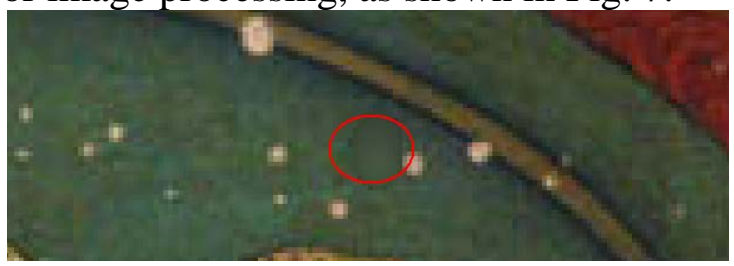

a_1 Effect image

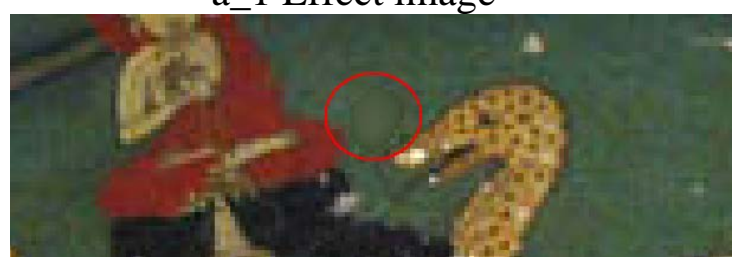

b_1. Effect image

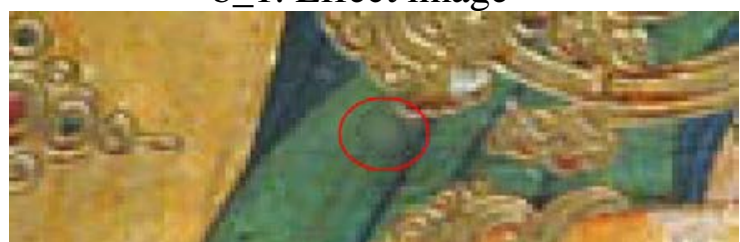

c_1. Effect image

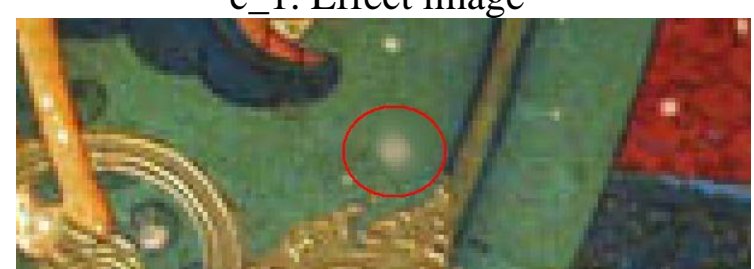

d_1. Effect image

Fig. $7.9 \times 9$ template

Experimental results show that when the image crack width is greater than 7 pixels, the size of the template with $7 \times 7$, repair bad results.

E. The different crack width of $11 \times 11$ template image processing, as shown in Fig. 8.

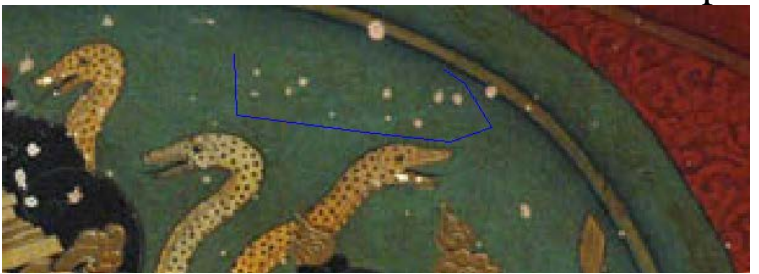

A. The original image

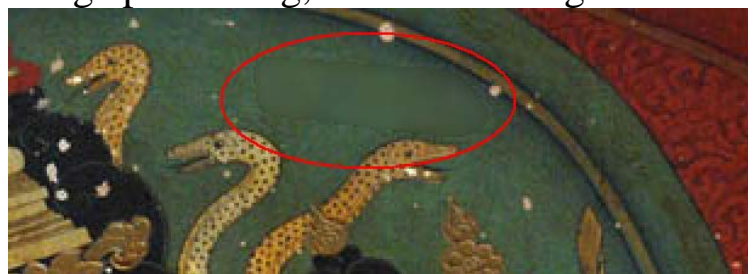

B. Effect image

Fig. $8.11 \times 11$ template 
The experimental result shows, when the template size is $11 \times 11$, have very good result for image repair, but the repaired area will be distortion at the same time in image repair.

The results of the experiment has proved that the use of median filter to repair the crack in the image, the template size should be less than $11 \times 11$.

The Relationship Between image background gray value of variations and repair results. To repair the crack in median filtering for the image, and the gray value of the image background change in different degree has a certain influence on repairing effect.

Selection the template and size is $5 \times 5$ to deal with the broken pieces, as shown in Fig. 9.
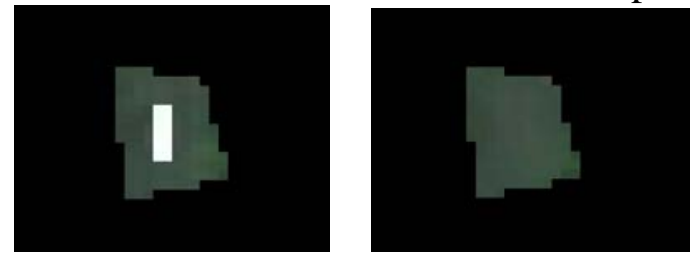

a. Two pixel width (white)
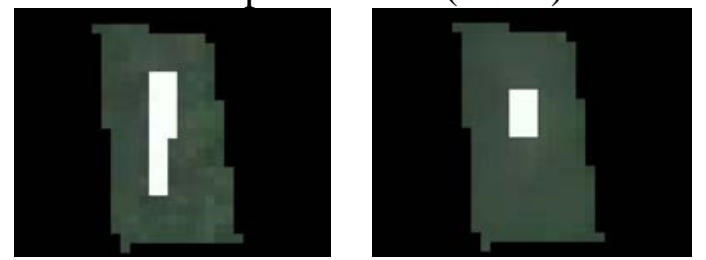

b. Three pixel width (white)

Fig. 9.Repair effect

Compare experiments results in Fig. 5b_1 with the results are shown in Fig. 9, as the picture broken crack gray value and image background gray value is too large in discrepancy, the median filter on the effect of image restoration is bad, as shown in Fig.9b.

Problems Arising From The Experiment And Improvement. Median filtering is sensitive to dot, line, spire details, after median filtering is used to repair the original image, and between the fixed area and the original image appear a similar bad black dot and white dot in sharp corner.

Through analysis, due to the nature of median filtering algorithm, for the current processing of eight neighborhood pixels to sort the value and obtain the mid value, When encountered in the angle of pixels, it will take the mid value of pixel values for zero(black).

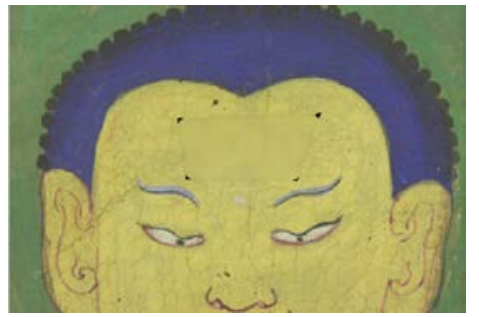

a

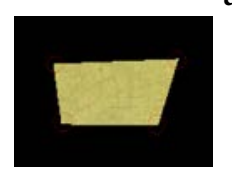

b

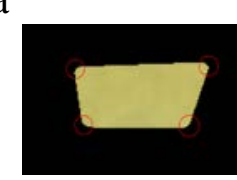

C

Fig.10. The first case

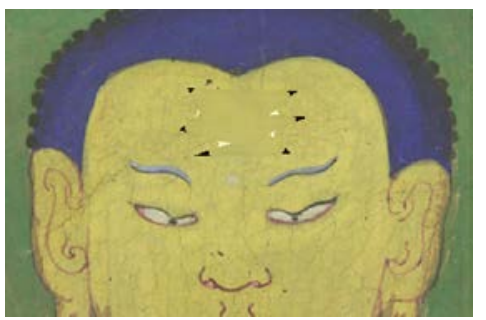

a

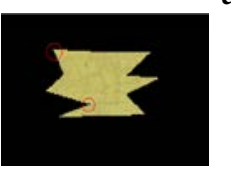

b

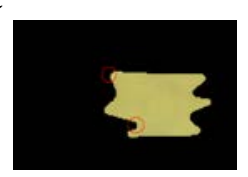

C
Fig.11. The second case

As shown in Fig. 10 and Fig. 11, the damage area of gray levels of pixels is smooth processing as the background color of the gray value. Fig. 10b and Fig. 10c, Fig.11b and Fig.11c comparison show that the extraction area of the vertex angle has been smoothed.

Solution:

First, making the gray value of pixels in Fig. 10b that has been smoothed, return to the gray value that before the image is processed. 
Second, making the gray value of pixels in Fig. 11b that has been smoothed, return to the gray value that before the image be processed.

\section{The Summary And Analysis Of Experimental Results}

The Process Of image Restoration. A. Extracting the cracks in the image area. In order to avoid critical areas of the image smooth fuzzy, we are adopt to the method of man-machine interaction, and we based on image edge, block to extract a part of the need to deal with. Taking ginput and line function of matlab, and using the man-machine interactive method to extract the crack area from image[7].

The concrete implementation:

a. Using ginput function to obtain the coordinates of the point;

b. Using line function in the two adjacent point of attachment, and until the end and starting point form a closed area;

c. Using roipoly function choose polygon area of gray image, the background to black, the area is white;

d. Extract the R, G, B component from the original image;

e. The original image of R, G, B component respectively with the polygon of image region do multiplication operation, and each component of the normalized, get the area of the image for repairing.

B. This experiment method is adopted to repair cracks area of the face that are extracted from the image, and extracting the area of the image has the same size with the original image. In addition to repair the crack area, other pixels in the image gray value is 0, as shown in Fig. 12.

C. Using the median filter and selecting the appropriate template, repairing the crack area of the face, as shown in Fig. 13.

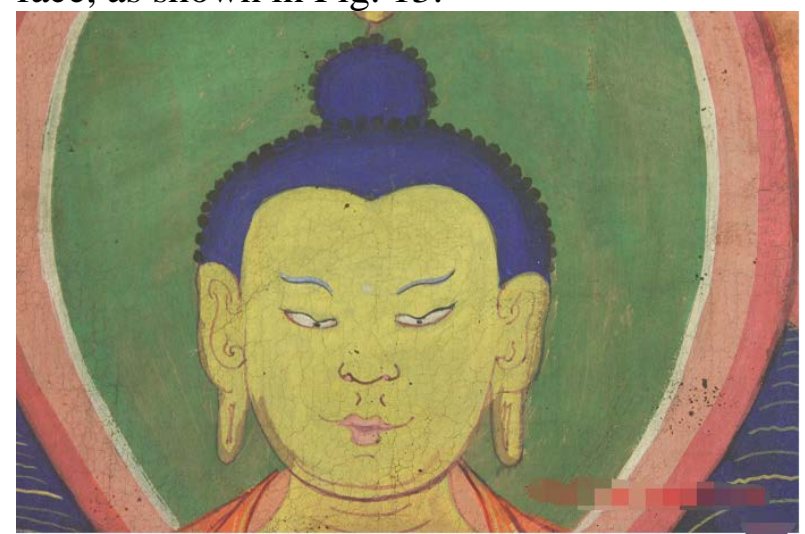

a

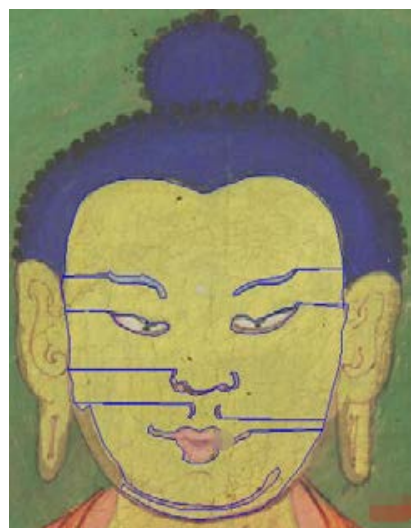

b

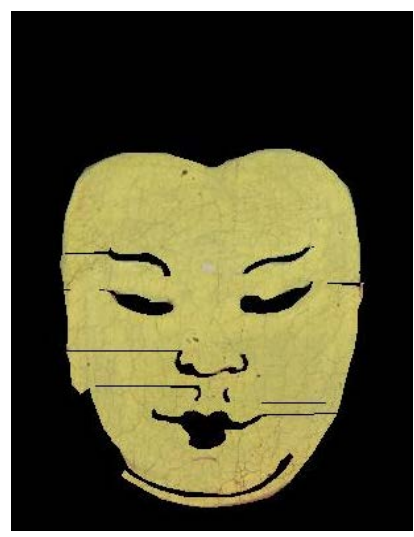

C

Fig. 12.Extract the crack area

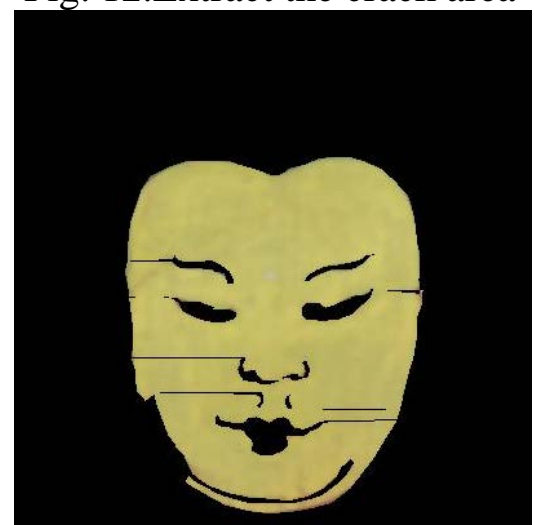

Fig. 13.Repair the crack area 
D. The repaired area and the area that be left after the crack area is extracted add together, finally fixed image, as shown in Fig. 14.
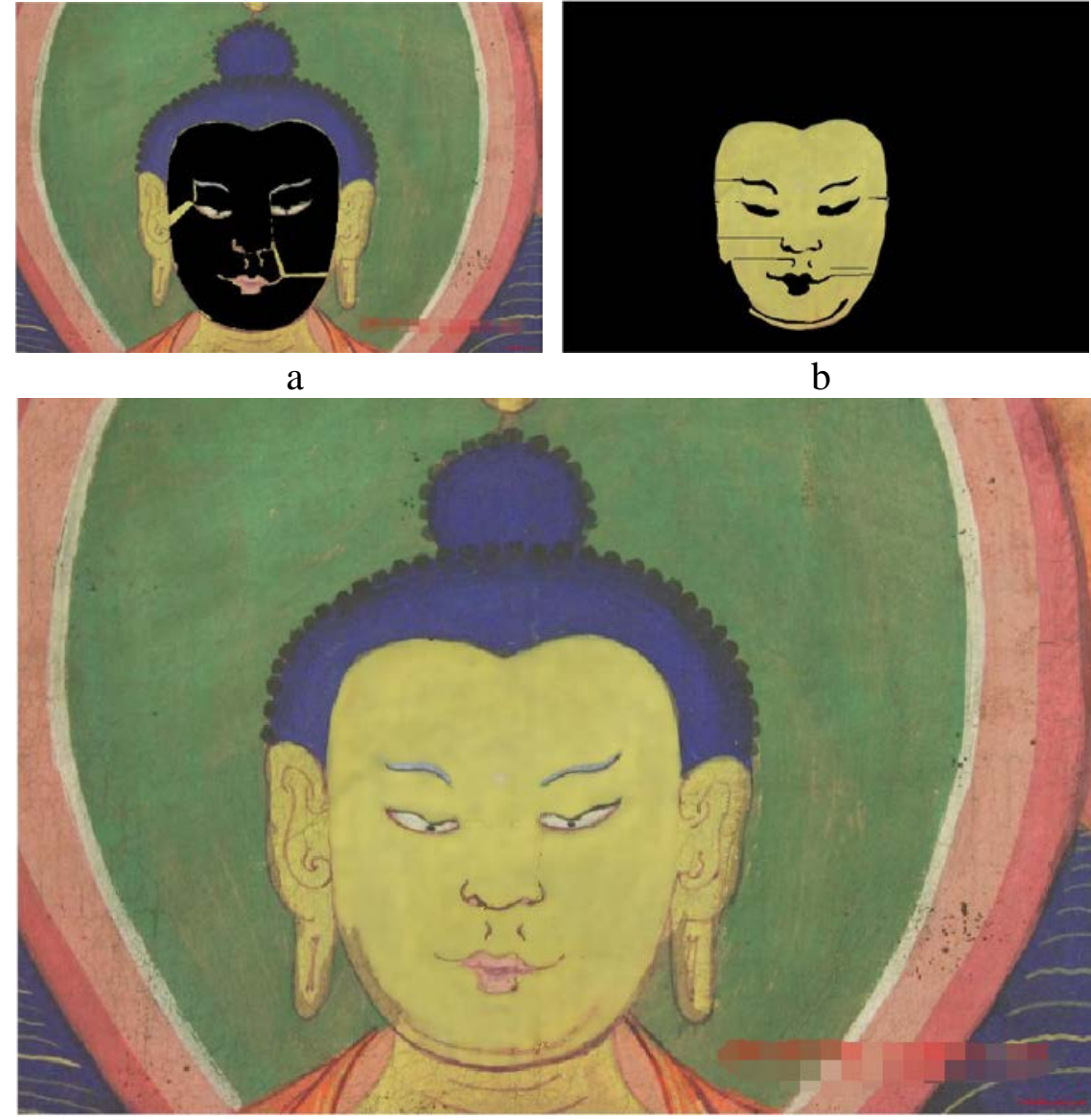

C

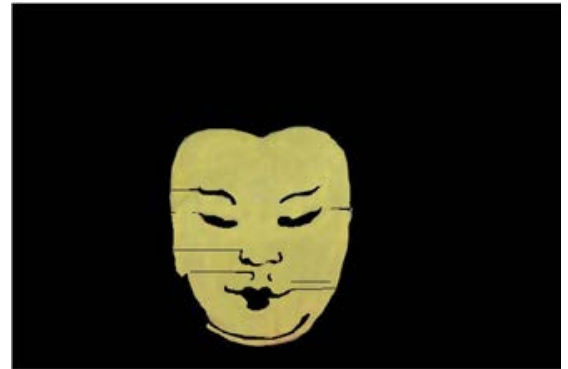

$\mathrm{b}$

Fig. 14.Effect image

Experiment results on the face show when the median filter that is used to repair the crack area of the image, which can get better repair results.

Analysis Of Image Restoration Method. The method are adopted to repair the crack area in the image in this study has some limitations:

A. It needs to get the crack area in the way of human-computer interaction, and it is difficult to operate, so we need professionals to operate.

B. According to the properties of the median filtering method, the image processing in the form of convolution, may cause blur the image and make the image key information loss; Therefore, when the crack area is extracted, and we should avoid the image edge and the key parts of the image.

\section{Conclusion}

In this paper, we are adopt to the median filter for image damaged crack repair, and it has good results on image restoration when select the square templates of median filtering and template size less than $11 \times 11$; As the image damage cracks is greater than 7 pixel width, it can't get good results when the median filter is adopted to repair. And experimental methods can't automatically extract the image damaged crack area, thus, reducing the efficiency of image processing. So when the median filter was applied to image restoration, the method should be improved further.

\section{Acknowledgements}

This work was supported by the National Natural Science Foundation of China (No.61162021, No.61561042), the personnel training program of State Ethnic Affairs Commission, and also was supported by Innovative Team Subsidize of Northwest University for Nationalities. 


\section{References}

[1] Four-Dimensional Technology, Xiaofeng Hu, Hui Zhao. Visual C + + / Matlab image processing and recognition application cases selected [M]. Beijing: People's Posts And Telecommunications Publishing House, 2004. In Chinese

[2] Ping Zhang. Based on the curvature and fast median filtering on image restoration method [J]. Anhui, Hefei University Of Technology, 2007. In Chinese

[3] Levin A, Zomet A, Weiss Y. Learning how to inpaint from global image statistics[C]//Computer Vision, 2003. Proceedings. Ninth IEEE International Conference on. IEEE, 2003: 305-312.

[4] Criminisi A, Pérez P, Toyama K. Region filling and object removal by exemplar-based image inpainting[J]. Image Processing, IEEE Transactions on, 2004, 13(9): 1200-1212.

[5] Kawai N, Yokoya N. Image inpainting considering symmetric patterns[C]//Pattern Recognition (ICPR), 2012 21st International Conference on. IEEE, 2012: 2744-2747.

[6] Liu H, Wang W, Xie H. Thangka image inpainting using adjacent information of broken area[C]//Proceedings of the International MultiConference of Engineers and Computer Scientists. 2008, 1

[7] Li Liu. Interested in based on Matlab image area extraction [J]. Journal of Sichuan province, southwest petroleum university, 2013. In Chinese

[8] Huaming Liu. Based on the sample piece of Thangka image restoration technology research [J]. Journal of gansu province, northwest university for nationalities, 2007. In Chinese

[9] Xiaobao Lu. Based on the breakage of the sample piece of thangka image restoration algorithm of improved [J]. Journal of gansu province, northwest university for nationalities, 2010. In Chinese 